\title{
UN NUEVO HORIZONTE DE PARTICIPACION DEL GOBIERNO CORPORATIVO DE LAS COOPERATIVAS EN EL PROCESO DE ADOPCION DE LAS NIIF EN NORTE DE SANTANDER.
}

\author{
A NEW HORIZON OF PARTICIPATION OF THE CORPORATE \\ GOVERNANCE OF THE COOPERATIVES IN THE PROCESS OF ADOPTION \\ OF THE IFRS IN NORTH OF SANTANDER.
}

\author{
PhD. Nubia Isabel Díaz Ortega*, MSc. Marisol Maestre Delgado**, Ruth Mayerly \\ Guerrero Jaimes", Ricardo Guerrero****
}

\begin{abstract}
* Universidad de Pamplona, Facultad de ciencias económicas y empresariales. Grupo de investigación CEYCON. Km 1 Vía Bucaramanga Ciudad Universitaria Pamplona - Norte de Santander. e- mail:ndiaz712@hotmail.com. Orcid Id: 0000-0001-7950-8664. e- mail: ruthguerrero@unipamplona.edu.co. Orcid Id: 0000-0002-2179-7691.
\end{abstract}

** Universidad de Pamplona, Facultad de ciencias económicas y empresariales. Grupo de investigación GISM. Km 1 Vía Bucaramanga Ciudad Universitaria Pamplona - Norte de Santander. e- mail: mmaestre24@ hotmail.com. Orcid Id: 0000-0002-0548-2710.

*** Universidad de Pamplona, Facultad de ciencias económicas y empresariales. Grupo de investigación GRAMMY. Km 1 Vía Bucaramanga Ciudad Universitaria Pamplona Norte de Santander. . e- mail:rivanguera1 @ hotmail.com. Orcid Id: 0000-0003-4859-

8921.

Resumen: El objetivo de la investigación será analizar la participación del gobierno corporativo en el proceso de adopción de las NIIF-PYMES en empresas del sector cooperativo de Norte de Santander, sustentándose teóricamente en autores como: (Vita ,2010), (Mantilla, 2015), (Rodríguez, 2014), (Estupiña, 2012), entre otros. Metodológicamente, se basará en el paradigma positivista, bajo un estudio cuantitativo, analítico, descriptivo y diseño mixto documental y de campo, no experimental con corte transeccional. La muestra está conformada por 73 empresas del sector cooperativo de Norte de Santander de la base de datos de la Superintendencia de Economía Solidaria. Para la recolección de los datos se diseñó un cuestionario que fue procesado bajo técnicas estadísticas. Los resultados indican una alta participación del gobierno corporativo en el proceso de adopción de las NIIF lo cual permite establecer un liderazgo del sector cooperativo en esta nueva cultura.

Palabras clave: Normas Internaciones, Gobierno Corporativo, Cooperativas, Proceso de Adopción. 


\begin{abstract}
The objective of the research will be to analyze the participation of corporate governance in the process of adoption of the IFRS-PYMES in companies of the cooperative sector of Norte de Santander, theoretically sustaining themselves in authors such as: (Vita, 2010), (Mantilla, 2015), (Rodríguez, 2014), (Estupiñan, 2012), among others. Methodologically, it will be based on the positivist paradigm, under a quantitative, analytical, descriptive study and mixed documentary and field design, not experimental with transectional cut. The sample consists of 73 companies in the cooperative sector of Norte de Santander from the database of the Superintendency of Solidarity Economy. To collect the data, a questionnaire was designed and processed using statistical techniques. The results indicate a high participation of the corporate government in the process of adoption of the IFRS which allows establishing a leadership of the cooperative sector in this new culture.
\end{abstract}

Key words: International Standards, Corporate Governance, Cooperatives, Adoption Process.

\section{INTRODUCCIÓN}

Hoy día, es un requisito para muchas empresas contar con un sistema contable adaptado a la normatividad internacional, las Normas Internacionales de Información Financiera juegan un papel importante como instrumento estratégico para insertarse en el mercado internacional, al realizar operaciones de exportación e importación, para hablar en los mismos términos financieros que la competencia internacional y dar la oportunidad de realizar la comparabilidad de sus cifras frente a las de sus competidores.

En consecuencia, la conversión exitosa hacia las NIIF implica un compromiso a largo plazo en las empresas, entre los principales autores que aportan su visión sobre el tema se encuentra (Rodríguez, 2014) indicando que se presentan cambios en el negocio, sistemas, procesos, contables, controles. No se trata meramente de la creación de un nuevo formato de estados financieros, sino que tiene que ver con la comunicación, educación y entrenamiento. Otros autores van más allá, para (Vásquez y Franco 2013), los costos de adoptar las NIIF envuelven aspectos de impacto a los preparadores, auditores y reguladores, induciendo una importante resistencia al cambio. El IASB toca temas de fondo que afectan el proceso de implementación de las NIIF como son la necesidad de formación y capacitación permanente de todos los autores involucrados, de nuevos sistemas de información de las empresas y cambios de la cultura contable de países con tradición legal como base para la emisión de principios de contabilidad generalmente aceptados.
Del examen anterior se advierte, que los cambios a realizar en las empresas durante el proceso de adopción tocan al sector cooperativo de Norte de Santander y deben ser liderados al interior de las mismas. En tal sentido, el compromiso de la alta dirección de las cooperativas juega un papel crucial en este proceso para llegar al éxito del mismo, lo que origina la necesidad de investigar en esta comunidad empresarial los efectos de la adopción y puesta en práctica de estas novedosas normas internacionales.

Por consiguiente, es pertinente realizar estudios enfocados en proporcionar información sobre las variables relativas al papel del gobierno corporativo de las cooperativas, así como, los aspectos referidos al proceso de adopción de las NIIF. En función de ello, el objetivo general del estudio se centró en analizar el papel del gobierno corporativo de las cooperativas de Norte de Santander durante el proceso de adopción de las NIIF.

\section{ANTECEDENTES}

A nivel mundial las empresas paulatinamente han enfrentado los retos de la globalización, que puede describirse según (Talla, 2010) como la internacionalización del conocimiento y de las actividades humanas. Es un fenómeno en el que se encuentran incluidos factores como el social, político, económico, cultural, entre otros, asî mismo, la contabilidad no podía quedarse atrás al ir reflejando los cambios ocurridos en las empresas. 
Como consecuencia lógica del proceso de globalización, la homogeneidad de la información contable y financiera se fue convirtiendo en la respuesta a la necesidad de una nueva interpretación de la información contable bajo los mismos parámetros, desde cualquier parte del mundo y en tiempo real, con el propósito de ayudar a los inversionistas y otros usuarios a tomar decisiones económicas. Cabe decir, que (Mallo y Pulido, 2004) afirman que la información derivada del registro de las transacciones debe ser homogénea, generar unos resultados de naturaleza similar y ofrecer una comparación de la situación patrimonial, cualquiera que sea el tipo de empresa y el país en que se divulgue.

Esta la misma forma, esta condición sumada a una serie de escándalos para los departamentos contables de grandes empresas a nivel mundial como señala (Estupiñan, 2012), maquillaje de balances y fraudes corporativos como consecuencia de la poca transparencia de la información financiera, dio luz por parte de organismos internacionales principalmente el Consejo de Normas Internacionales de Contabilidad a las Normas Internacionales de Información Financiera. Dentro de los objetivos del IASB se encuentra desarrollar un conjunto de estándares globalmente aceptados, comprensibles y de alta calidad que mejoren la transparencia y comparabilidad de la información financiera mediante el uso de un lenguaje común que puede ser utilizado por todos los países. Asimismo, reflejan la realidad económica y financiera de las organizaciones, por lo tanto, son dinámicas y cambiantes. Según (Mejía, 2003) las NIIF lograrán mejorar la calidad de los estados financieros, se ganará en comparabilidad y credibilidad, las NIIF serán una herramienta para crear un clima de confianza alrededor de la información financiera en el mundo.

En este orden de ideas, en el 2013 de acuerdo con un análisis realizado por Deloitte sobre la aplicación de las NIIF en 174 países del mundo, las estadísticas indican que las NIIF se permiten, exigen su utilización o están convergiendo con ellas en 123 países, es decir, en una gran mayoría. Por lo tanto, Colombia inmerso en ese proceso de globalización inició los procesos de conversión a NIIF de sus empresas, en la actualidad se encuentra en la era de la aplicación definitiva, siguiendo el cronograma que estableció inicialmente la denominada ley de convergencia contable Ley
1314 de julio 13 de 2009 que después fue modificado en reiteradas ocasiones hasta el año 2013 en donde se visualiza la hora definitiva de migración hacia NIIF a partir del año 2014. Las empresas fueron clasificadas en tres grandes grupos, primer grupo NIIF plenas, segundo grupo NIIF PYMES y tercer grupo contabilidad simplificada.

En tal sentido, (Rodríguez, 2014) define las NIIF para PYMES como un conjunto de estándares de información financiera simplificado en función de las necesidades de información de los usuarios y en la relación costo beneficio de la obtención de la información financiera. Se regulan por el Decreto 3022 de diciembre de 2013, que estableció dentro de las condiciones para pertenecer a dicho grupo no ser principalmente emisores de valores ni entidades de interés público, además no cumplir ninguna de las siguientes condiciones: ser subordinada o sucursal de una compañía extranjera que aplique NIIF plenas, ser subordinada o matriz de una compañía nacional que deba aplicar NIIF plenas, ser matriz, asociada o negocio conjunto de una o más entidades extranjeras que apliquen NIIF plenas o realizar importaciones o exportaciones, realizar importaciones o exportaciones que representen un $50 \%$ de sus operaciones de compras o ventas, tener más de 200 empleados y activos totales inferiores a 30.000 salarios mínimos.

Al mismo tiempo, la Superintendencia de Economía Solidaria precisó este mismo ámbito de aplicación al definir en la Circular No. 005 de 2014 cuales son las empresas que pertenecen al grupo 2 , coincidiendo al establecer que se trata de entidades que no cumplan los requisitos del artículo 1 del Decreto 2784/ 2012 ni con los requisitos del capítulo 1 del marco técnico normativo del Decreto 2706/2012, no tener portafolios de terceros administradores de sociedades comisionistas de bolsas y el número de trabajadores y los activos totales se calculan por el promedio de los últimos doce meses.

Para abordar el tema de la transición es pertinente indicar que el Decreto 3022 en su artículo 3 estableció unas fechas claras para las empresas que integran el grupo 2 de NIIF PYMES debían realizar la preparación obligatoria durante al año 2014, a partir del 1 de enero de 2015 debían comenzar el periodo de transición de contabilidad local a contabilidad bajo NIIF y el año de 
aplicación de primeros estados financieros con NIIF PYMES no comparativos a 31 de diciembre de 2015. Al respecto, la Superintendencia de Economía Solidaria emitió orientaciones para las empresas ubicadas en el Grupo 2 en la Circular Externa No 08 de 2015, indicando que el estado financiero de apertura se presentará ante la Superintendencia durante el mes de septiembre de 2015.

Es decir, el grupo 2 de NIIF para PYMES realizó su periodo de transición, sin embargo, al hablar de transición implica hacer referencia a una serie de cambios en los sistemas de reportes gerenciales, contables, indicadores de desempeño y finanzas corporativas, entre otros. Para (Mantilla, 2013), la transición a NIIF no puede hacerse de manera improvisada, porque serán muchos los cambios, por ejemplo, en el sistema de contabilidad, en los informes financieros, en las comunicaciones dentro de la organización, en las funciones de auditoría. Sin embargo, los efectos de la transición son más amplios el mismo autor señala que se trata de un proceso integrado en tres áreas importantes personas, tecnologías y procesos. En Europa según (García, 2013), durante el periodo de transición hubo muchas opiniones y preocupaciones por parte de los responsables de los sectores contables, impositivos y tecnología de las empresas y en el primer año de aplicación las empresas reconocían que las normas incrementaban la aproximación a la realidad económica, pero, al mismo tiempo, eran muy complejas de aplicar y requerían mucho trabajo adicional.

En la actualidad, el departamento Norte de Santander y especialmente la ciudad de Cúcuta vive en estos momentos un periodo de ajuste en su economía según el Informe de Gestión 2014 de la Cámara de Comercio los empresarios han puesto su mirada en el mercado interno y en otros destinos diferentes a Venezuela, la ciudad tiene una de las tasas más altas de desempleo del país con un $12 \%$ y una informalidad del $71 \%$. De esto se desprende la importancia del sector cooperativo que hace parte del sector formal y según estadísticas de la Superintendencia de Economía Solidaria a 31 de diciembre de 2015 en el departamento de Norte de Santander existían 321 entidades del sector cooperativo, que presentan cifras relevantes tenían contratados 1.293 empleados, con un valor en activos de $\$ 697.286 .808 .475$ e ingresos por un valor de $\$ 454.840 .181 .775$
Por otro lado, las empresas del sector cooperativo de Norte de Santander realizaron los procesos de adopción, en el periódico Contacto. Coop edición 35 de agosto de 2013 el sector mostró su interés por el tema de las NIIF mostrando las ventajas que su aplicación traería al sector cooperativo siendo conscientes que deben enfrentarse al reto y a la obligación de adaptar sus sistemas contables a las NIIF.

\subsection{Gobierno corporativo en las cooperativas en Colombia}

La gobernabilidad corporativa se refiere al sistema mediante el cual las organizaciones sean del sector público o privado son dirigidas y controladas. Para la (OCDE, 1999) la estructura del gobierno corporativo especifica la distribución de derechos y responsabilidades entre los diversos actores de la empresa. El gobierno corporativo siguiendo a la (Vita, 2010) es la manera como la empresa protege los intereses de accionistas y otros deudores. Se refiere a la responsabilidad de la gerencia incluyendo directores y miembros de la Junta Directiva. La Superintendencia de Economía Solidaria ha adaptado estos conceptos en su guía de buen gobierno, deja por sentado que el gobierno corporativo es el proceso mediante la cual el consejo de administración guía a una organización en el cumplimento de su misión corporativa, además el gobierno corporativo en las cooperativas la conforman la asamblea, el gerente y la junta de vigilancia.

Inicialmente, el IASB dirigió sus esfuerzos a crear una norma para la pequeñas y medianas entidades, normas que según (Estupiñan, 2012), serian normas que se ajustarían al clima organizacional, a los requerimientos e información y a la disponibilidad de los recursos para adoptar e implementar dichas normas. Estas normas fueron publicadas en julio de 2009 por el IASB en un documento final denominado NIIF para PYMES que consta de 35 secciones organizadas por temas y con ejemplos ilustrativos de los estados financieros con la respectiva información a revelar. Luego, en Colombia este documento fue acogido por el direccionamiento estratégico del Consejo Técnico de la Contaduría para esencialmente las entidades que no negocian en bolsa o manejen recursos del público, es así, como más tarde el Decreto 3022 de diciembre de 2013 estableció los requisitos para pertenecerá este grupo y finalmente 
actualizado por el Decreto 2267 de 2014.

Las empresas del sector cooperativo en Colombia, se encuentran reguladas por la Ley 454 de 1998 que determina el marco conceptual, sus principios orientadores, características, organismos, entidades $\mathrm{y}$ en general los normas que rigen al sector. Con base en el artículo 22 de esta ley la Superintendencia de Economía Solidaria que es el máximo organismo de control expidió la Circular externa básica contable y financiera 004 de 2008 que modificó las bases contables y financieras de las cooperativas armonizándolas a NIIF, estableciendo un compendio normativo que tiene como propósito construir entidades solidarias confiables.

Para (Estupiñan, 2013), las pequeñas y medianas empresas deben ajustar las NIIF a su clima organizacional, a sus requerimientos de información y a su disponibilidad de recursos para adoptar e implementar estas normas. En su criterio, la adopción tiene alcances en el sistema contable e información financiera, procesos y sistemas de información, recursos humanos y comunicación interna y externa. Esto significa que en las empresas se empezaron dar cambios importantes a raíz de la adopción, la estandarización de la contabilidad comprende aspectos novedosos para las empresas en los procesos, actividades, modelos de negocio, controles organizacionales y marco normativo (Cáceres et al., 2015).

El proceso de adopción comprende varias etapas (Mantilla, 2015) la planeación y ejecución. La implementación de las NIIF requiere de un proyecto bien organizado, con cronograma, presupuesto, calidad y seguimiento. También, Chorafas divide la planeación en un primer hito y la etapa de ejecución en dos hitos importantes la salida a aprueba y la real. Para (Rodríguez, 2014) las etapas son Fase 1 Diagnóstico, Fase 2 Diseño y Planeación, Fase 3 Desarrollo de Soluciones, Fase 4 Implementación y Fase 5 Implementación Posterior.

\section{METODOLOGIA}

La investigación se califica como una investigación de tipo descriptiva, la misma se orientó a analizar las políticas de administración de las cooperativas durante el proceso de adopción de las NIIF en empresas cooperativas de Norte de Santander Colombia. Para empezar, el diseño es no experimental, de campo, transversal, la recolección de datos se realizó en un único momento, obteniendo de esta forma una sola medición.

Luego, para la recolección de los datos se usó un cuestionario, que fue sometido al juicio de expertos, el cuestionario comprendió aspectos sobre el gobierno corporativo y el proceso de adopción de las NIIF, el total de ítems fue de 31 valorados en la escala de Lickert. (Siempre, Casi siempre, a veces, casi nunca y nunca). La confiabilidad del instrumento se determinó con el método Alfa Crombach, arrojando como resultado rtt: 082, lo que determina que el instrumento es altanamente confiable.

Después, el cuestionario validado se aplicó en las cooperativas siendo las unidades informantes, los contadores, jefes financieros, y funcionarios de la administración. Para la determinación de la muestra se tomó como población a las cooperativas y entidades del sector de la base de datos de la Superintendencia de Economía Solidaria, para la muestra se consideraron 73 cooperativas.

\section{Tabla 1. Clasificación de entidades del sector solidario tomadas como muestra.}

\begin{tabular}{lc}
\hline \multicolumn{1}{c}{ Descripción } & Cant. \\
\hline Fondos & 13 \\
$\begin{array}{l}\text { Cooperativas } \\
\begin{array}{l}\text { Otros (Asociaciones, Fundaciones y } \\
\text { uniones mutuales) }\end{array}\end{array}$ & 55 \\
\hline
\end{tabular}

Fuente: Elaboración propia, (2018)

La muestra se encuentra ubicada en las ciudades más importantes del departamento de Norte de Santander y se encuentra distribuida así: Cúcuta 40, Pamplona 18 y Ocaña 15.

Para la recolección de los datos se diseñó como instrumento un cuestionario y los resultados fueron procesados en el software SSPS 23 aplicando técnicas estadísticas. Para terminar, los resultados se presentaron con estadísticas descriptivas y medidas de tendencia central, además se presenta la discusión de los datos. 


\section{RESULTADOS Y DISCUSIÓN}

De los resultados obtenidos en la investigación se han seleccionados los más importantes, al indagar sobre como la administración en el proceso de adopción de las NIIF realizó una planeación estratégica, se pudo evidenciar según la Gráfica 1 que arrojó que un alto número de cooperativas con un $66 \%$, realizó una planeación estratégica, mientras que un $24 \%$ mediamente y el $10 \%$ no se involucró en este proceso. Para (Zamudio, 2014), la planeación estratégica representa el cimiento del proceso "pues esta herramienta promete y cumple a cabalidad con cada uno de los lineamientos que se deben tener en cuenta para que la implementación de nuevos proyectos en una organización tengan éxito" También, el proceso de adopción de estar plasmado en un plan que debe ser presentado para aprobación de la alta administración (Mantilla, 2015).

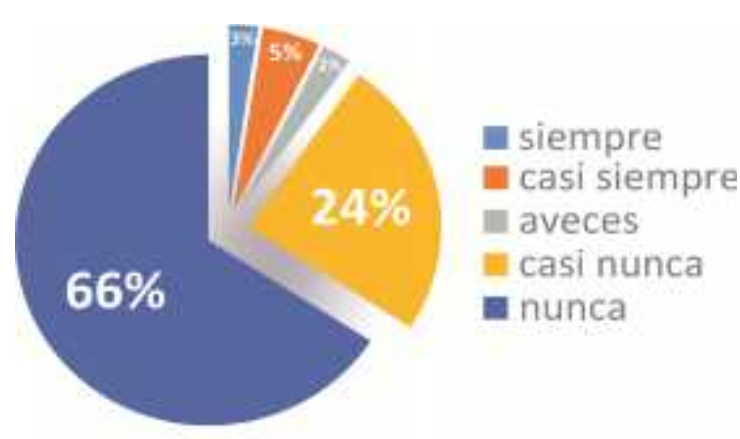

Fig. 1. Planeación estratégica

La Grafica 2 presenta que el Consejo de administración aprobó la aplicación de las nuevas políticas contables bajo NIIF en un $85 \%$ y el $15 \%$ de entidades lo realizó muy escasamente. Es de resaltar la importancia del Consejo de Administración al estar atentos a los detalles del proceso de adopción de las NIIF como son las determinación de las políticas contables, pues en Turquía, los planteamientos de (Misirliogli et al., 2013) concluyeron que las cuestiones derivadas del gobierno corporativo significó una restricción significativa para la adopción exitosa de las NIIF. Al respecto, resultados de investigaciones realizadas en Irán por (Hassan et al., 2014) detectaron que dentro de las causas para que la adopción de las NIIF no sea percibida como meramente simbólica se encuentra como necesaria la reforma del gobierno corporativo de las empresas. Sin embargo, en países de África subsahariana, (Kosarkoska y Mircheska, 2012) mostraron que la mayoría de los países habían adoptados prácticas de buena gobernanza empresarial y encontraron una relación positiva entre el índice de gobierno corporativo y las prácticas contables.

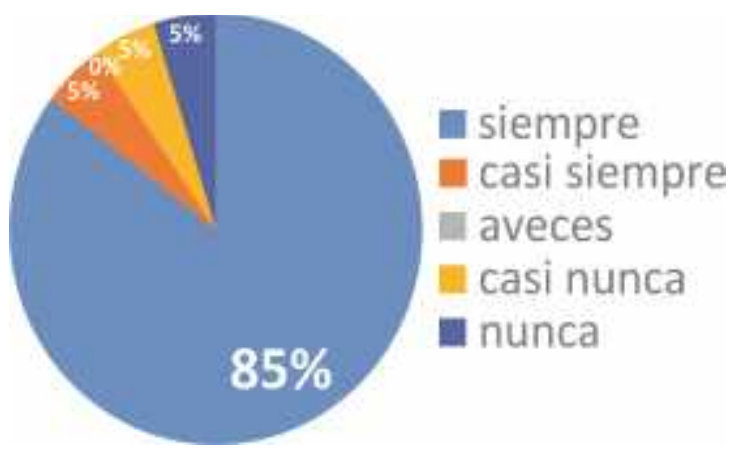

Fig. 2. Políticas contables

Dentro del cuestionario se le pidió a los encuestados que informaran si el proceso de adopción cuenta con la aprobación del responsable principal es decir del representante legal, a lo cual respondieron que un $85 \%$ tiene la aprobación de la gerencia y un $15 \%$ que escasamente la tenían. Este resultado coincide con otras investigaciones realizadas en empresas argentinas y chilenas que muestran que el comportamiento discrecional de la gerencia en la transición a las NIIF (Verón y Marcolini, 2016), es decir, el representante legal juega un papel destacado en el proceso de adopción de las NIIF.

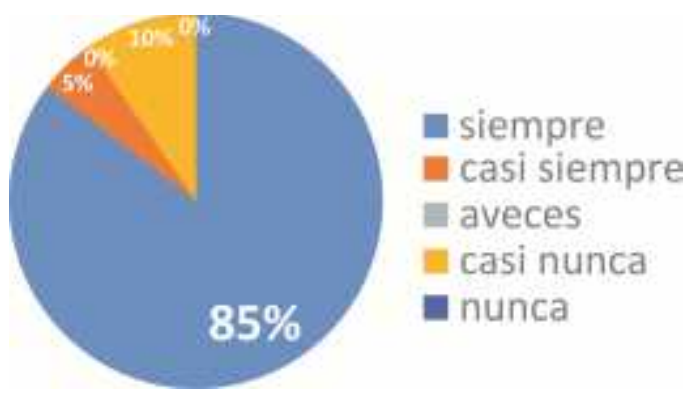

Fig. 3. Aprobación de representante legal 
Como se aprecia en la Gráfica 4, para la muestra el $71 \%$ reconoce el liderazgo estratégico del gerente, para $15 \%$ un gran participación y solo un $14 \%$ escasamente hay intervención del gerente en la adopción de las NIIF. En indagaciones realizadas en el país muestran los patrones que todo gerente de empresa como líder debe asumir en el manejo contable y financiero, con respecto a la nueva contabilidad internacional, concluyendo que se debe forjar una cultura hacia las NIIF para impedir costos mayores que bien podrán evitarse, (García y Dueñas, 2016). Por ende, la alta gerencia debe familiarizarse con el proyecto de adopción y sus impactos, pues requiere el liderazgo desde el nivel máximo de la organización para preparar una conversión exitosa (Lesmes, 2013), (Jánica, 2012), (López, 2012), (Orive, 2015).

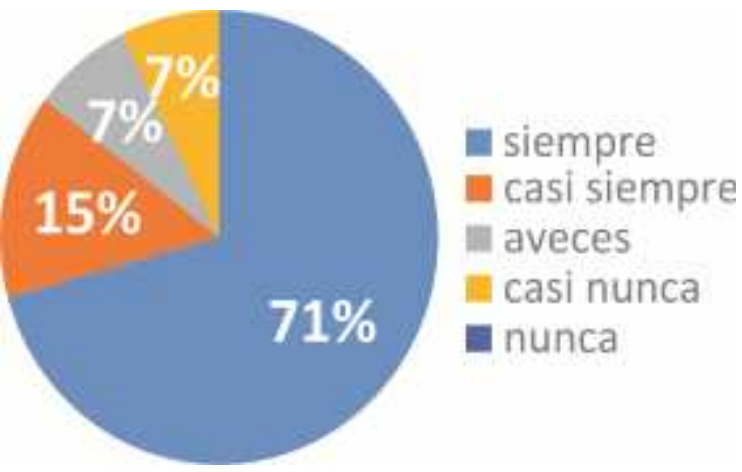

Fig. 4. Liderazgo estratégico de la gerencia.

Actualmente, en muchos casos el proceso de adopción se ha dejado en manos del contador, la responsabilidad de la adopción se ha dejado solamente en cabeza de los contadores públicos que presten sus servicios a las pymes (Ocampo y Astudillo, 2015), no obstante, a nivel empresarial se trata de un proyecto que suele involucrar a toda la organización y que requiere de liderazgo y cultura organizacional. (Estupiñan, 2013) expone que las NIIF no es una actividad solo contable relacionada con las cifras, sino que tiene un alcance muy grande hacia toda la organización incluyendo los niveles directivos distintos a la parte financiera. Los resultados demuestran en el sector de las cooperativas que la alta dirección ha participado activamente del proceso de adopción de las NIIF con $56 \%$, un $22 \%$ casi siempre, un $17 \%$ a veces y solo un $5 \%$ nunca ha participado.

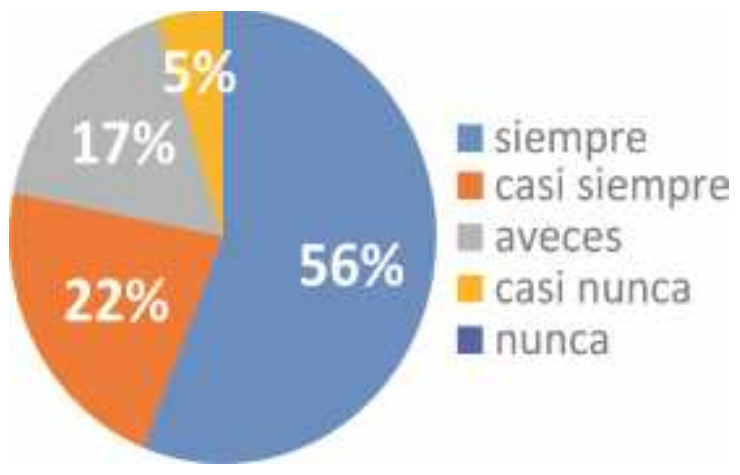

Fig. 5. Participación de alta dirección.

\section{CONCLUSIONES}

Las NIIF representan un cambio trascendental que debe ser liderado a través de la mediación de gerentes y órganos de dirección que transformen la cultura organizacional adecuadamente, el objetivo de la presente investigación fue analizar la participación del gobierno corporativo durante el proceso de adopción delas NIIF en las empresas del sector cooperativo e Norte de Santander.

De los resultados obtenidos se pudo derivar que el gobierno corporativo del sector cooperativo de Norte de Santander, ha sido muy positivo y ha realizado un gran acompañamiento al proceso de adopción de las NIIF. El gobierno corporativo ha participado de manera permanente y responsable en todas las etapas del proceso de adopción como fueron su planificación estratégica, la definición de las políticas contables, el liderazgo del proceso y una gran participación de la alta dirección. Por último, se observa que la alta participación del gobierno corporativo ha sido la guía para tener un proceso adopción de las NIIF sin mayores contratiempos y tropiezos.

\section{REFERENCIAS}

Deloitte IAS Plus. (2013). Use of IFRS by jurisdicción. Consultado el 5 de mayo de 2015. Disponible en :

http://www.iasplus.com/en/resources/ifrstopics/use-of-ifrs.

Cámara de Comercio de Cúcuta. (2015). Informe de Gestión 2014. Consultado el 28 de mayo de 2015. Disponible en http://www.cccucuta.org.co/media/Documentos linforme de_gestion_2014.pdf 
Estupiñan, R. (2012). Estados Financieros Básicos según las NIC Y NIIF. Ecoe Ediciones. Bogotá.

Estupiñan, R. (2013). NIC/NIIF. Transición y adopción en la empresa. Implementación por primera vez de las NIIF Plenas o FULL y de las NIIF para las PYMES. Ecoe Ediciones. Bogotá.

Revista Cuadernos de Contabilidad. Vol. 14 numero 36. Universidad Pontificia Javeriana, Bogotá.

García, I. (2013).Introducción a las NIIF. Ejercicios y casos reales en la web. Editorial Alfaomega Grupo Editor México.

García, S. \& Dueñas, R (2016). Los aspectos cualitativos de las PYMES y los nuevos retos de la gerencia frente a las NIIF. Revista Academia y Virtualidad Universidad Nueva Granada. Vol. 9 (2).

García, S, Dueñas, R \& Acuña, H. (2016). Las NIIF una forma de educar a los gerentes en la gestión de la pyme. Revista Ciencia y Poder Aéreo. Vol. 11 (1). Revista Científica de la Escuela de Postgrados de la Fuerza Aérea Colombiana.

Hassan, E., Rankin, M., \& Wei, L. (2014). The Development of Accounting Regulation in Iraq and the IFRS Adoption Decision: An Institutional Perspective. The International Journal of Accounting, Volume 49, Issue 3, September 2014, Pages 371-390

Kosarkoska, D. \& Mircheska, I. (2012). The Main Process In the International Financial Reporting at the Begining of 21st Century. Procedia Social and Behavioral Sciences, Volume 44, 2012, Pages 241-249

Lesmes, R. (2013). La globalización contable llegó al país: bienvenidos a los IFRS/NIIF. Revista Portafolio, Ene 2013.

López, A. (2012I. Vence plazo voluntario para ajustarse a las IFRS. Revista Portafolio; Bogotá (Mar 9, 2012).
Ley 1314 Convergencia Contable (2010). Diario Oficial de Colombia, 47.409. Julio 13, 2010.

Decreto 3022 (2013).Diario Oficial de Colombia, 49.016. Julio 27, 2013.

Mantilla, S. (2013). Entendiendo lo básico. Los IFRS/NIIF bien desde la primera vez. Editorial Ecoe Ediciones Ltda. Bogotá.

Mantilla, S. (2015). Estándares/Normas Internacionales de Información Financiera. (IFRS/NIIF). Editorial Ecoe Ediciones Ltda. Bogotá.

Mallo C y Pulido A. (2004). Las Normas Internacionales de Información Financiera. Editorial Thomson. Madrid.

Mejía, E. (2003) Normas internacionales de contabilidad. Universidad del Quindío. Armenia.

Misirlioglu, I., Tucker, J., \& Yukselturk, O. (2013). Does Mandatory Adoption of IFRS Guarantee Compliance? The International Journal of Accounting, Volume 48, Issue 3, September 2013, Pages 327-363

Rendón, B, Montaño, E. y Gaitán, G. Las NIIF y su impacto en las cooperativas de Colombia a 31 de diciembre de 2011. Revista Cuadernos de Administración de la Universidad del Valle.

Rodríguez, C. (2014). Estado de Situación Financiera de Apertura para NIIF PYMES. ¿Qué hacer y cómo hacerlo? Instituto Colombiano de Estudios Fiscales. Medellín.

Ocampo, P, \& Astudillo, R, (2015). Retos y alternativas en la implementación de las NICNIIF en las Pymes colombianas. Revista Magazín Empresarial. Vol. 11 (26). Pág. 1119. Universidad Santiago de Cali.

Orive, L. 2015. La importancia del liderazgo en la empresa. Instituto Nacional de Contadores. Consultado el 21 de abril de 2017. Disponible 
en: http://www.incp.org.co/la-importancia-delliderazgo-en-la-empresa/

Portafolio. Alerta por atraso en las normas NIIF. Consultado el 1 de mayo de 2015. Disponible en:

http://www.portafolio.co/economia/normascontabilidad-mundial

Superintendencia de Economía Solidaria. Tabla de entidades supervisadas. Consultado el 09 de junio de 2015. Disponible en: www.supersolidaria.gov.co.

Superintendencia de Economía Solidaria. Guía para el buen gobierno. Consultado el 29 de abril de

2017.Disponibleen:https://www.supersolidaria .gov.co/sites/default/files/public/data/guia_bu en_gobierno_marzo_2013.pdf

Talla, Sabino. (2010) La globalización y la armonización contable. Revista Quipukamayuc. Facultad de Ciencias Contables. México. Consultado el 6 de junio de 2015.Disponible en http://sisbib.unmsm.edu.pe/bibvirtual/publicac iones/quipukamayoc/1999/primer/global.htm

Vásquez, R. y Franco, W. (2013). El ABC de las NIIF. Guía básica de preguntas y respuestas para la implementación de las IFRS-NIIF. Legis Editores S.A. Bogotá.

Vásquez, R. y Franco, W. (2014) Aplicación por primera vez de las NIIF. NIIF plenas y NIIF pymes. Guía práctica. Legis Editores S.A. Bogotá.

Verón, C. \& Marcolini, S. (2016). El balance de transición a NIIF en las empresas argentinas y chilenas: la relación entre los ajustes obligatorios y los ajustes voluntarios. Revista Fundamentos. Disponible en: http://revista.eco.unrc.edu.ar/index.php/fundam entos/article/view/21/11. Consultada el: 6 de marzo de 2017.
Zamudio, Y. (2014). La planeación estratégica un aspecto clave en la implementación de las normas internacionales de información financiera niif. Tesis de Grado. Universidad Militar Nueva Granada. Bogotá, Colombia. 